\title{
Solute transport modelling with the variable temporally dependent boundary
}

\author{
PINTU DAS*, AFFREEN AKHTER and MRITUNJAY KUMAR SINGH \\ Department of Applied Mathematics, Indian Institute of Technology (Indian School of Mines), \\ Dhanbad 826004, India \\ e-mail: pintudas62@gmail.com; affreen92@gmail.com; drmks29@rediffmail.com
}

MS received 16 February 2017; revised 8 June 2017; accepted 9 June 2017; published online 7 February 2018

\begin{abstract}
In this present study, analytical and numerical solutions are obtained for solute transport modelling in homogeneous semi-infinite porous medium. The dispersion coefficient is assumed to be initial dispersion and velocity is assumed to be temporally dependent with initial seepage velocity. Also, the concept of dispersion is directly proportional to the square of the seepage velocity used for finding the solution. Initially, the domain is not solute free. At one end of the domain, source concentration with the effect of different temporally dependent functions taken into account. The concentration gradient assumed to be zero due to no mass flux at other end of the domain. Laplace Transform Technique is used to obtain the exact solution, whereas Explicit Finite Difference method is used for approximate solution. The different types of temporally dependent velocity are used for the graphical representation of the solution. The accuracy of the solution explored by the Relative error analysis.
\end{abstract}

Keywords. Dispersion; advection; porous medium; relative error; solute.

\section{Introduction}

Groundwater pollution concerns various theoretical and experimental studies of chemical transport through porous medium. Transport processes are similar to those for rigid porous medium and include advection, dispersion and sorption. The most traditional method used to model the transport of solute in the groundwater is the advection dispersion equation. This equation includes various terms which describe the physical process such as advection, dispersion, linear isotherms, decay parameter, etc. Kumar [1] developed the analytical solution of one-dimensional advection-dispersion equation with unsteady flow against dispersion in finite porous media using Laplace transform technique. Van Kooten [2] employed the solution of onedimensional solute transport equation with spatially varying velocity field and dispersion coefficients in the presence of kinetically absorbing solute in porous media. Leij et al [3] discussed analytical solution of advection dispersion equation for solute transport in uniform porous media with steady one or two-dimensional flow using Green function method. Zeng and Bennett [4] studied the analytical and numerical solutions for the solute transport modelling in groundwater flow with multi-layered aquifer system. Quezada et al [5] developed the analytical solution for coupled multi-dimensional, multi-species advection-dispersion

*For correspondence equations with first order kinetic reaction network. De Smedt et al [6] established an exact analytical solution for solute transport in streams from an instantaneous injection source. The solution includes the effect of exchange with transient storage zones, modelled by first order rate equation. Zhan et al [7] established an analytical solution for two-dimensional solute transport equation in aquifer-aquitard system by maintaining rigorous mass conservation at the aquifer-aquitard interface. Qiu et al [8] described the analytical solution for solute transport model equation with spatially dependent flow velocity and solute dispersion using Generalized Integral Transform Technique. Gao et al [9] discussed an analytical solution of solute transport model with asymptotic scale-dependent dispersion function in finite heterogeneous porous media. The analytical solution solved by using Laplace transform technique and extended power series method. You and Zhan [10] discussed semianalytical solutions for the one-dimensional solute transport in a finite domain with time-dependent sources and distance-dependent dispersivities. Also, existing solution developed for spatial dispersivity through a laboratory column test. Deng et al [11] discussed an analytical solution for solute transport in multi-layered porous media using generalized integral transform technique with arbitrary boundary conditions. Singh and Das [12] discussed an analytical and numerical solution for one-dimensional scale-dependent solute dispersion in heterogeneous porous medium. The accuracy of the result explored by Root mean square 
method. Majdalani et al [13] established solute transport in periodical heterogeneous porous medium with scale dependent dispersion at high degree of heterogeneity variation. Gharehbaghi [14] presented numerical solution of one-dimensional advection-dispersion equation in semi-infinite domain by using differential quadrature method. Sanskrityayn and Kumar [15] explored the analytical solution of the solute transport modelling in heterogeneous porous media subjected to the instantaneous and continuous injected sources using Green's function method. Falta and Wang [16] established the semi-analytical solution of onedimensional advection-dispersion equation with matrix diffusion process. The derived semi-analytical solution by assuming the low permeability in semi-infinite domain.

The motivation of this study is to derive an analytical and numerical solution of one-dimensional advection-dispersion equation in semi-infinite aquifer with the effect of linear isotherm. The Laplace Transform Technique is adopted to derive exact solution, and explicit finite difference method for the approximated solution. The different types of transient dependent input source taken into consideration at one end of the domain. The dispersion coefficient is assumed to be initial dispersion and velocity is directly proportional to the initial seepage velocity taken into account. The different types of temporally dependent velocity pattern used to predict the concentration pattern. The accuracy of the solution gives the better agreement between the analytical and numerical one.

\section{Mathematical formulation}

Transport of solute in groundwater is modelled by onedimensional advection-dispersion equation. In this model, the transport of solute in one-dimensional advection-dispersion equation with solid-liquid inter phase described as (Batu [17])

$$
\frac{\partial c}{\partial t}+\frac{(1-n)}{n} \frac{\partial F}{\partial t}=\frac{\partial}{\partial x}\left(D \frac{\partial c}{\partial x}-U c\right)
$$

where $D\left[L^{2} T^{-1}\right]$ is the dispersion coefficient, $c\left[M L^{-3}\right]$ is the volume averaged dispersing solute concentration in the liquid phase, $F\left[M L^{-3}\right]$ is the volume averaged dispersing solute concentration in the solid phase, $U\left[L T^{-1}\right]$ is the seepage velocity, $x[L]$ is the direction of flow, $t[T]$ is the time and $n$ is porosity.

Consider mathematical expression for linear isotherm as

$$
F=k_{d} c
$$

where $k_{d}$ is the distribution coefficient.

Using Eq. (2) in Eq. (1), we have

$$
R \frac{\partial c}{\partial t}=\frac{\partial}{\partial x}\left(D \frac{\partial c}{\partial x}-U c\right)
$$

where $R=1+\frac{(1-n)}{n} k_{d}$ is the retardation factor.

For solving the governing advection-dispersion equation we required the initial and boundary conditions. Initially, the linear expression of initial concentration with angular frequency is taken into account. Mathematical expression of initial condition is as follows

$$
c(x, 0)=c_{i}+w \quad x>0, t=0 .
$$

At one end of the domain, different types of temporally dependent source concentration are taken into consideration. Due to no mass flux at the other end of the domain, concentration gradient is assumed to be zero.

$$
\begin{gathered}
c(0, t)=\frac{c_{0}}{2}(1+\sec w t) \quad x=0, t>0 \\
\frac{\partial c}{\partial x}=0, \quad x \rightarrow \infty, t>0
\end{gathered}
$$

where $w\left[T^{-1}\right]$ is the angular frequency [18].

Now, using the concept that dispersion is directly proportional to the initial dispersion coefficient and velocity is directly proportional to the initial seepage velocity, we have

$$
U(x, t)=U_{0} f(m t), \quad D=D_{0} .
$$

Using Eq. (7) in Eq. (3), we have

$$
R \frac{\partial c}{\partial t}=\frac{\partial}{\partial x}\left(D_{0} \frac{\partial c}{\partial x}-U_{0} f(m t) c\right)
$$

To conveniently use Laplace transform technique we introduced the new space dependent and time dependent transformations as follows [19]

$$
\begin{aligned}
z & =\frac{x}{f(m t)} \\
\text { and } \quad T & =\int_{0}^{t} \frac{1}{f(m t)^{2}} d t
\end{aligned}
$$

Using Eqs. (9) and (10) in Eq. (8), we have

$$
R \frac{\partial c}{\partial T}=D_{0} \frac{\partial^{2} c}{\partial z^{2}}-U_{0} f(m t)^{2} \frac{\partial c}{\partial z} .
$$

Again using the concept of dispersion and seepage velocity, we have

$$
\begin{gathered}
D=a U^{2} \Rightarrow D_{0}=a U_{0}^{2} f(m t)^{2} \\
u_{0}=\frac{D_{0}}{a U_{0}}=U_{0} f(m t)^{2} .
\end{gathered}
$$

Using Eq. (12a) and (12b) in Eq. (11), we have 


$$
R \frac{\partial c}{\partial T}=D_{0} \frac{\partial^{2} c}{\partial z^{2}}-u_{0} \frac{\partial c}{\partial z} .
$$

According to the above transformations, our initial and boundary conditions can be written as

$$
\begin{gathered}
c(z, 0)=c_{i}+w \quad z>0, T=0 \\
c(0, T)=\frac{c_{0}}{4}\left(4+w^{2} T^{2}\right) \quad z=0, T>0 \\
\frac{\partial c}{\partial z}=0 \quad z \rightarrow \infty, T>0
\end{gathered}
$$

Now, introducing a new transformation

$$
c(z, T)=k(z, T) \exp \left(\frac{u_{0} z}{2 D_{0}}-\frac{u_{0}^{2} T}{4 R D_{0}}\right) .
$$

Using Eq. (16), partial differential Eq. (13) transformed with initial and boundary conditions (14)-(15b) and solved using Laplace Transform Technique, the desired analytical solution is as follows (see Appendix):

$$
c(z, T)=\left(\frac{c_{0}-c_{i}-w}{2}\right) F(z, T)+\frac{w^{2} c_{0}}{2} G(z, T)+H(z, T)
$$

where

$$
F(z, T)=\operatorname{erfc}\left(\frac{z R-u_{0} T}{2 \sqrt{D_{0} R T}}\right)+\exp \left(\frac{u_{0} z}{D_{0}}\right) \operatorname{erfc}\left(\frac{z R-u_{0} T}{2 \sqrt{D_{0} R T}}\right)
$$

$$
\begin{aligned}
G(z, T)= & \left(\frac{1}{2 \alpha}\right)\left(\alpha T^{2}-\frac{z T}{2} \sqrt{\frac{R}{D_{0}}}+\frac{z}{4 \alpha^{2}}-\frac{z T}{2}+\frac{z^{2}}{4 \alpha} \sqrt{\frac{R}{D_{0}}}\right) \\
& \times \operatorname{erfc}\left(\frac{z R-u_{0} T}{2 \sqrt{D_{0} R T}}\right) \\
& +\left(\frac{1}{2 \alpha}\right)\left(\alpha T^{2}+\frac{z T}{2} \sqrt{\frac{R}{D_{0}}}-\frac{z}{4 \alpha^{2}}+\frac{z T}{2}+\frac{z^{2}}{4 \alpha} \sqrt{\frac{R}{D_{0}}}\right) \\
& \times \exp \left(\frac{u_{0} z}{D_{0}}\right) \operatorname{erfc}\left(\frac{z R+u_{0} T}{2 \sqrt{D_{0} R T}}\right) \\
& -\left(\frac{1}{2 \alpha}\right) \frac{z}{\alpha} \sqrt{\frac{T}{\pi}} \mathrm{e}^{-\frac{z^{2} R}{4 I D_{0}}}
\end{aligned}
$$

$$
H(z, T)=c_{i}+w
$$

2.1 The effect of velocity with decay parameter at one end of the boundary

Consider linear expression of velocity with time at one end of the domain [1]

$$
c(0, t)=(1-w U t)^{\frac{q}{w}} \quad x=0, t>0
$$

where $w\left[T^{-1}\right]$ is the angular frequency, $U\left[L T^{-1}\right]$ is the seepage velocity and $q\left[T^{-1}\right]$ is the decay parameter.
By using the above transformations and applying Laplace Transform Technique, we get the desired analytical solution as

$$
c(z, t)=\frac{\left(1-c_{i}-w\right)}{2} F_{1}(z, T)-\frac{q_{0}}{2} F_{2}(z, T)+F_{3}(z, T)
$$

where

$F_{1}(z, T)=\operatorname{erfc}\left(\frac{z R-u_{0} T}{2 \sqrt{D_{0} R T}}\right)+\exp \left(\frac{u_{0} z}{D_{0}}\right) \operatorname{erfc}\left(\frac{z R+u_{0} T}{2 \sqrt{D_{0} R T}}\right)$

$$
\begin{gathered}
F_{2}(z, T)=\left(u_{0} T-z R\right) \operatorname{erfc}\left(\frac{z R-u_{0} T}{2 \sqrt{D_{0} R T}}\right) \\
+\left(u_{0} T+z R\right) \exp \left(\frac{u_{0} z}{D_{0}}\right) \operatorname{erfc}\left(\frac{z R+u_{0} T}{2 \sqrt{D_{0} R T}}\right) \\
F_{3}(z, T)=c_{i}+w .
\end{gathered}
$$

\subsection{The effect of zero order production with velocity}

The solute can grow in liquid and solid phase. The growth of solute expressed by the decay parameter. The effect of zero order production with velocity is taken into account at one end of the domain. Mathematically, it can be expressed as

$$
c(0, t)=\frac{\gamma}{U} t \quad x=0, t>0
$$

where $\gamma=\frac{\gamma_{0}}{f(m t)}$.

By using the above transformations and applying Laplace Transform Technique, we get the desired analytical solution as

$$
c(z, t)=\frac{\gamma_{0}}{2 u_{0}^{2}} G_{1}(z, T)-\frac{\left(c_{i}+w\right)}{2} G_{2}(z, T)+G_{3}(z, T)
$$

where

$$
\begin{aligned}
G_{1}(z, T)= & \left(u_{0} T-z R\right) \operatorname{erfc}\left(\frac{z R-u_{0} T}{2 \sqrt{D_{0} R T}}\right) \\
& +\left(u_{0} T+z R\right) \exp \left(\frac{u_{0} z}{D_{0}}\right) \operatorname{erfc}\left(\frac{z R+u_{0} T}{2 \sqrt{D_{0} R T}}\right)
\end{aligned}
$$

$$
G_{2}(z, T)=\operatorname{erfc}\left(\frac{z R-u_{0} T}{2 \sqrt{D_{0} R T}}\right)+\exp \left(\frac{u_{0} z}{D_{0}}\right) \operatorname{erfc}\left(\frac{z R+u_{0} T}{2 \sqrt{D_{0} R T}}\right)
$$

$$
G_{3}(z, T)=c_{i}+w .
$$




\section{Numerical solution}

Modelling of solute transport in groundwater through aquifer is a serious concern these days. In the context of pollution dispersion in groundwater, advection-dispersion equation has been solved analytically and numerically using appropriate initial and boundary conditions. Chen et al [20] derived implicit and explicit finite difference methods for solving the fractional reaction--sub-diffusion equation.

To solve numerically the Eqs. (13)-(15b) in semi-infinite medium converted into finite medium. In order to solve by the finite difference technique, the semi-infinite medium is changed into a finite medium by using suitable transformation expressed as

$$
X=1-\exp (-z)
$$

By using above transformation Eqs. (13)-(15b) can be written as

$$
R \frac{\partial c}{\partial T}=D_{0}(1-X)^{2} \frac{\partial^{2} c}{\partial X^{2}}-\left(D_{0}+u_{0}\right)(1-X) \frac{\partial c}{\partial X}
$$

and corresponding initial and boundary condition can be written as

$$
\begin{gathered}
c(X, 0)=c_{i}+w \quad X>0, T=0 \\
c(0, T)=\frac{c_{0}}{4}\left(4+w^{2} T^{2}\right) \quad X=0, T>0 \\
\frac{\partial c}{\partial X}=0 \quad X=1, T>0 .
\end{gathered}
$$

Now using explicit finite difference approximation with forward time and central space forward difference scheme, Eqs. (32)-(34b) can be approximated as

$$
\begin{gathered}
c_{i, j+1}=c_{i, j}+\frac{D_{0}}{R}\left(1-X_{i}\right)^{2}\left[c_{i+1, j}-2 c_{i, j}+c_{i-1, j}\right] \frac{\Delta T}{\Delta X^{2}} \\
-\frac{\left(D_{0}+u_{0}\right)}{2 R}\left(1-X_{i}\right)\left[c_{i+1, j}-c_{i-1, j}\right] \frac{\Delta T}{\Delta X} \\
c_{i, 0}=c_{i}+w, \quad i>0 \\
c_{0, j}=\frac{c_{0}}{4}\left(4+w^{2} T_{j}^{2}\right) \quad j>0 \\
c_{M, j}=c_{M-1, j} \quad j>0 .
\end{gathered}
$$

The boundary condition in Eqs. (21) and (26) can be approximated as

$$
c_{0, j}=1-q_{0} u_{0} T_{j} \quad j>0
$$

and

$$
c_{0, j}=\frac{\gamma_{0}}{u_{0}} T_{j} \quad j>0
$$

where the subscripts $i$ and $j$ refer to space and time, respectively, and $\Delta T$ is the time increment and $\Delta X$ is the space increment in Eq. (35).

The space variable $X$ and time domain $T$ are discretized by rectangular grid points $\left(X_{i}, T_{j}\right)$ with mesh size $\Delta X$ and $\Delta T$, respectively. Hence

$$
\begin{gathered}
X_{i}=X_{i-1}+\Delta X \quad i=1,2,3 \ldots M, X_{0}=0, \Delta X=0.03 \\
T_{j}=T_{j-1}+\Delta T \quad j=1,2,3 \ldots N, T_{0}=0, \Delta T=0.001
\end{gathered}
$$

where $M$ and $N$ are integers, and $c_{i, j}$ is the contaminant concentration at a point for the space domain $X_{i}$ with time domain $T_{j}$.

\section{Stability analysis}

The stability analysis of explicit finite difference method for the one-dimensional advection-dispersion equation is solved by using the matrix method [12]. Using finite difference scheme, the governing partial differential equation can be rearranged as

$$
\begin{gathered}
c_{i, j+1}=c_{i-1, j}(\alpha+\beta)+c_{i, j}(1-2 \alpha)+c_{i+1, j}(\alpha-\beta) \\
\text { where } \alpha=\frac{D_{0} \Delta T}{R \Delta z^{2}}, \beta=\frac{u_{0} \Delta T}{2 R \Delta z} .
\end{gathered}
$$

The matrix of the form of Eq. (41) can be written as

$$
[c]^{j+1}=A[c]^{j}
$$

where $A$ is the matrix which contain all the constant.

By applying Gerschgorin circle method, the stability criteria for time step is

$$
\Delta T \leq \frac{R \Delta z^{2}}{D_{0}} .
$$

\section{Accuracy}

The comparison of the analytical and numerical solution is more systematic and consistent by adopting the criterion of Relative Percentage (RP) error between the analytical solution and the numerical solution at all grid points. In this paper, RP error is used to compare the numerical result to analytical one. The relative percentage error is given by

$$
\text { Relative Error }=\left|\frac{\Delta c}{c_{\text {analytical }}}\right|
$$

where

$$
\Delta c=c_{\text {analytical }}-c_{\text {numerical }}
$$




\section{Result and discussion}

The two different time dependent velocity forms are considered for the prediction of the contaminant concentration in the finite length of the domain. These time dependent velocity patterns mathematically can be written as

1. $f(m t)=\sqrt{(1-\sin (m t))}$

$$
T=\frac{1}{m}(\sec m t+\tan m t-1)
$$

2. $f(m t)=\exp (-m t)$

$$
T=\frac{1}{2 m}[\exp (2 m t)-1]
$$

The analytical and numerical solutions for the present problem are computed for the given set of input data [15] $c_{i}=0.1, c_{0}=1.0, U_{0}=0.5 \mathrm{~km} /$ year, $D_{0}=0.03 \mathrm{~km}^{2} /$ year, $\gamma_{0}=0.01, q_{0}=0.1 /$ year, $m=0.01 /$ year, $n=0.25$ (Gravel) and 0.40 (Clay).

The graphical representations are predicted in the finite length of the domain $0 \leq x \leq 0.7$ for different input source contaminants. The graphical representations are made for the different time interval $t=1$ and 2 (year), which describe the concentration pattern in aquifer (i.e. Gravel) and aquitard (i.e. Clay) in the finite length of the domain. Figure 1 depicts the contaminant concentration pattern for the unsteady sinusoidal form of velocity for the analytical solution given in Eq. (17) with minimum range of total porosity in different geological formations. It is observed from figure 1 that contaminant concentration pattern increases with time in both geological formations but it follows the decreasing nature with respect to distance. Also, we observed that the clay medium attains maximum concentration values as compared with the gravel medium but both the media follow the same values of concentration after covering some distance. Figure 2 predicts the exponential decreasing velocity

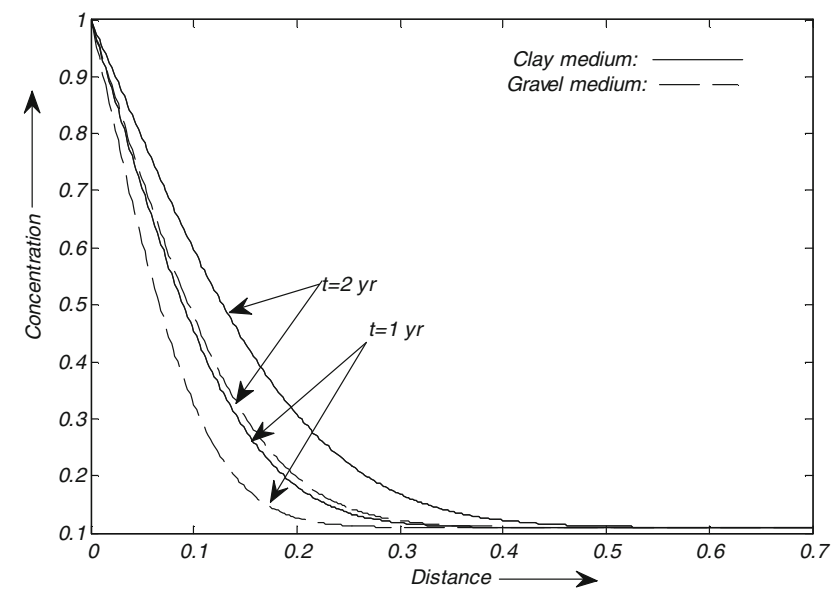

Figure 1. Concentration distribution for sinusoidal velocity pattern for boundary condition $\frac{c_{0}}{2}(1+\sec w t)$.

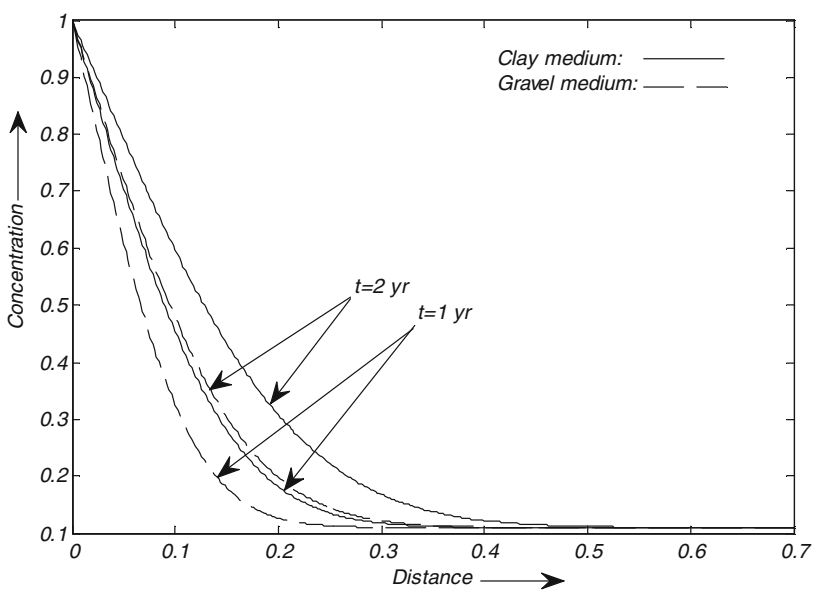

Figure 2. Concentration distribution for exponentially decreasing velocity pattern for boundary condition $\frac{c_{0}}{2}(1+\sec w t)$.

pattern for 1 and 2 years for the gravel and clay media with their minimum range of total porosity. It is observed that the concentration values increase with respect to time but decrease with respect to space, and ultimately both the patterns follow their minimum harmless concentration at the end of the domain. The clay medium follows the higher value of concentration as compared with the gravel medium. Figure 3 predicts for the comparison between analytical and numerical one for the different geological formations with their minimum range of total porosity for the time period of 2 years. Initially, analytical solution as compared to numerical one attains the same value and after covering some distance it attains the more value as compared with numerical one. Ultimately, both the patterns follow the same value at the end of the domain. The clay medium attains the more concentration value at each of the positions for the analytical and numerical one as compared to the gravel medium, but both the patterns follow the minimum harmless concentration at the end of the domain. Similarly, figure 4 predicts the

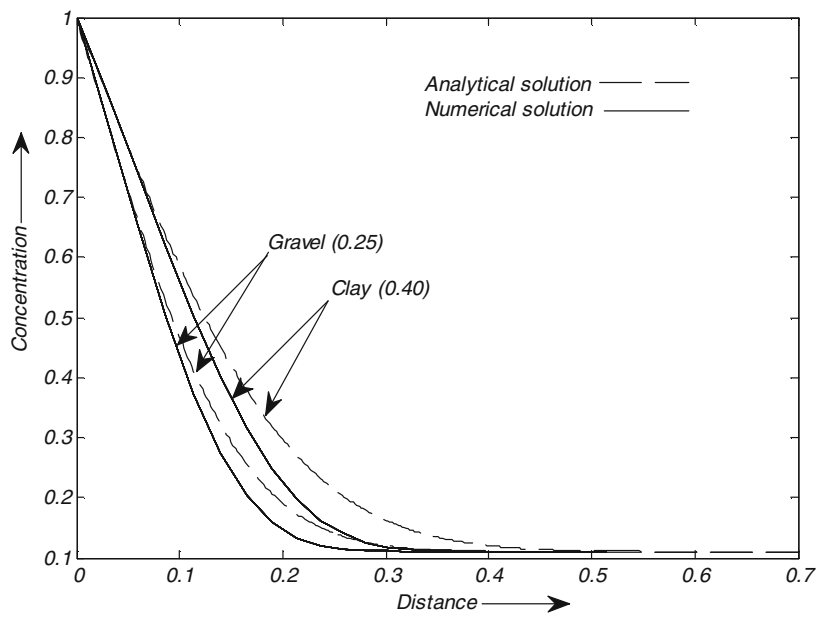

Figure 3. Comparison of concentration distribution for sinusoidal velocity pattern for boundary condition $\frac{c_{0}}{2}(1+\sec w t)$. 


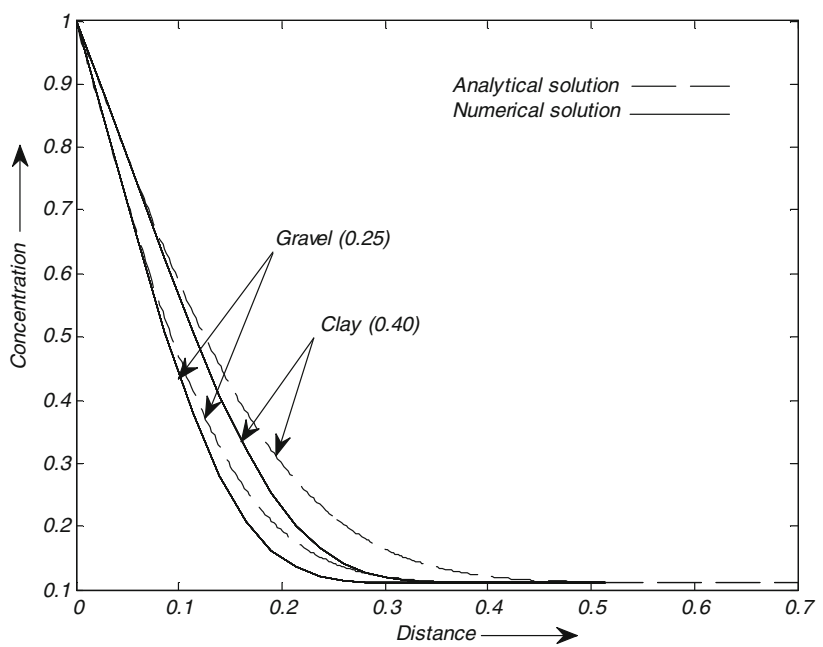

Figure 4. Comparison of concentration distribution for exponentially decreasing velocity pattern for boundary condition $\frac{c_{0}}{2}(1+\sec w t)$.

comparison between the analytical and numerical one for the exponential decreasing velocity pattern for the time period 2 years. In this velocity pattern, initially both the solutions attain the same value of concentration for different geological formations with their minimum range of porosity. After covering some distance, numerical solution attains more concentration value as compared with analytical one but at the end of the domain both the solutions follow the same concentration value. Figures 5, 6, 7 and 8 predict the concentration patterns for the analytical and numerical solution corresponding to the boundary condition defined in Eq. (21). Figure 5 predicts the comparison between the clay and gravel medium with their minimum range of porosity for the sinusoidal velocity pattern. From this figure, we observed that the clay medium attains the maximum value of the concentration at each of the position as compared to the gravel medium for different time period but both the medium attain their

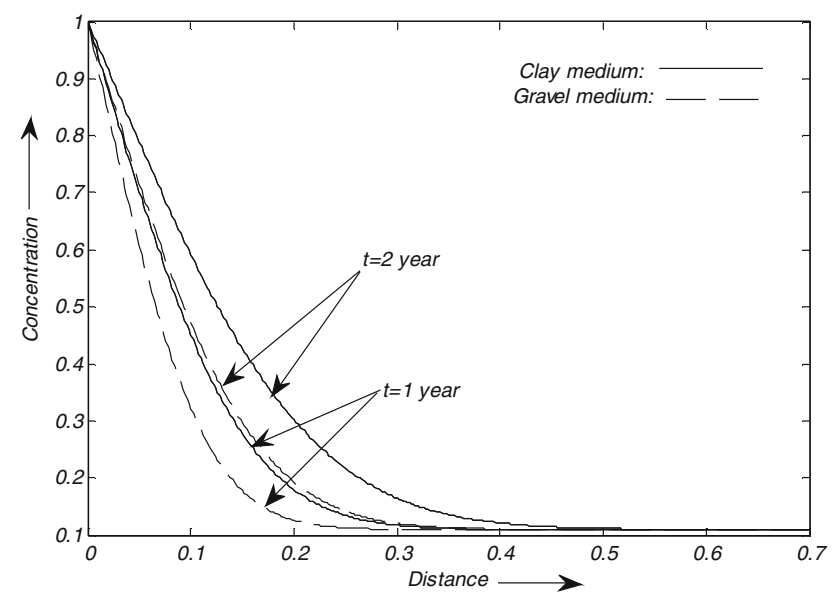

Figure 5. Concentration distribution for sinusoidal velocity pattern for boundary condition $(1-w U t)^{\frac{q}{w}}$.

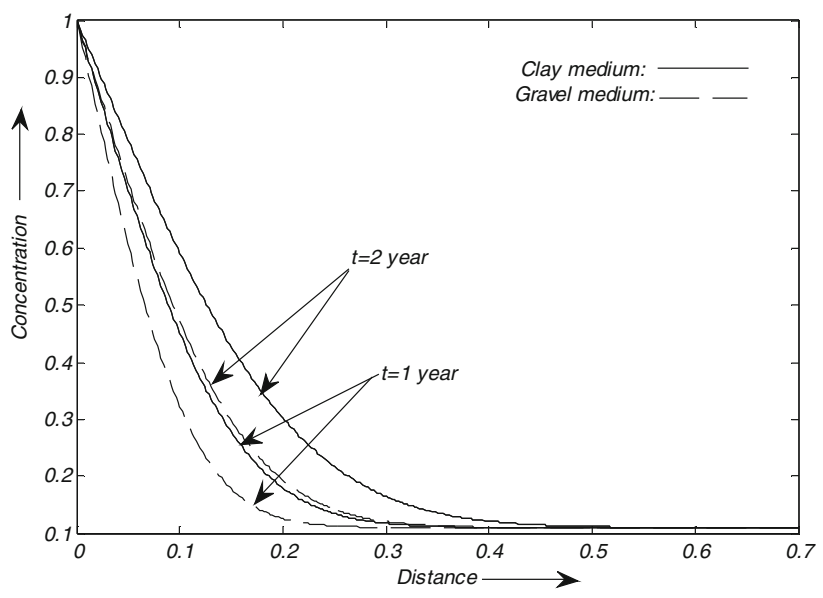

Figure 6. Concentration distribution for exponentially decreasing velocity pattern for boundary condition $(1-w U t)^{\frac{q}{w}}$.

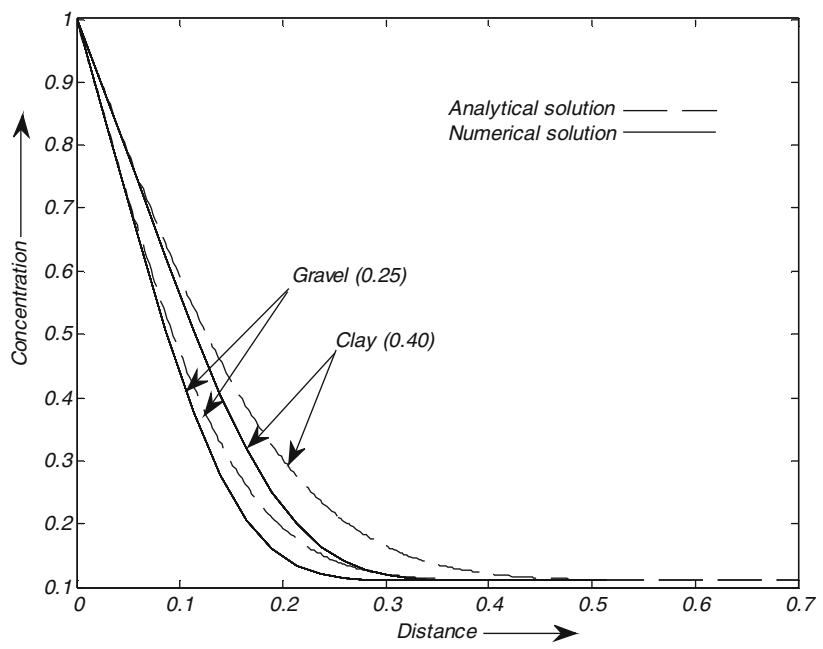

Figure 7. Comparison of concentration distribution for sinusoidal velocity pattern for boundary condition $(1-w U t)^{\frac{q}{w}}$.

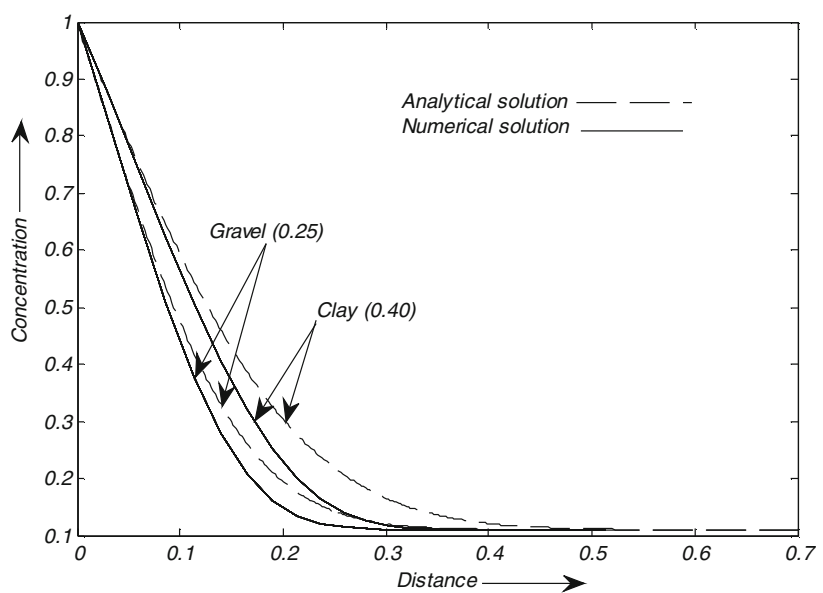

Figure 8. Comparison of concentration distribution for exponentially decreasing velocity pattern for boundary condition $(1-w U t)^{\frac{q}{w}}$. 
minimum harmless concentration at the end of the domain. Similarly, figure 6 predicts for the exponential decreasing velocity pattern, which follows the same concentration pattern as in figure 5. The comparison between the analytical and numerical one for the time period of 2 years is predicted in figure 7 with the minimum range of the porosity for clay and gravel medium. Initially, analytical and numerical solutions attain the same concentration values but after covering some distance, analytical solutions attain the higher concentration values as compared with numerical one but at the end of the domain both the patterns follow thier minimum harmless concentration. Similar concentration pattern along with the analytical and numerical one is shown in figure 8 for exponentially decreasing velocity pattern. From figure 8 , we observed that the clay medium attains higher concentration value both in analytical and numerical one as compared to gravel medium. Figures 9, 10, 11 and 12 predict the concentration patterns for the analytical and numerical solution corresponding to the boundary condition defined in Eq. (26). Figure 9 predicts for the clay and gravel medium with their minimum range of porosity for the time period of 1 and 2 years. As time increases, concentration values also increase at each of the position but they decrease with respect to distance and go to their minimum harmless concentration at the end of the domain. Clay medium attains higher concentration values as compared with the gravel medium. Similarly, figure 10 predicts the exponential decreasing velocity pattern for the clay and gravel medium. Figure 11 predicts the comparison between the analytical and numerical solutions for the time period of 2 years. The analytical solution attains the same concentration values as in numerical one, but beyond some distance it attains the higher value of concentration. Both the solutions attain thier minimum harmless concentration values at the end of the domain. Similar type of concentration pattern for the exponential decreasing velocity pattern for the gravel and clay medium is observed from figure 12. Comparison between the

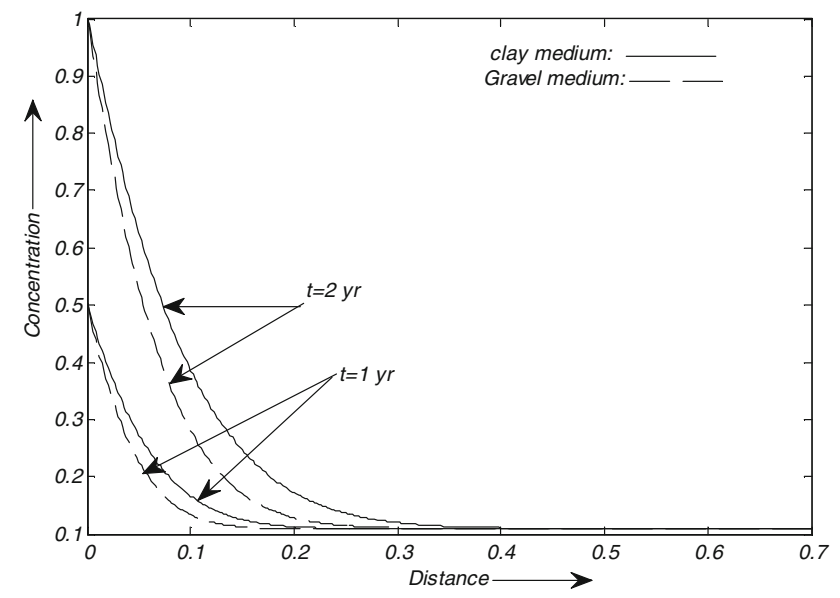

Figure 9. Concentration distribution for sinusoidal velocity pattern for boundary condition $\frac{\gamma}{U} t$.

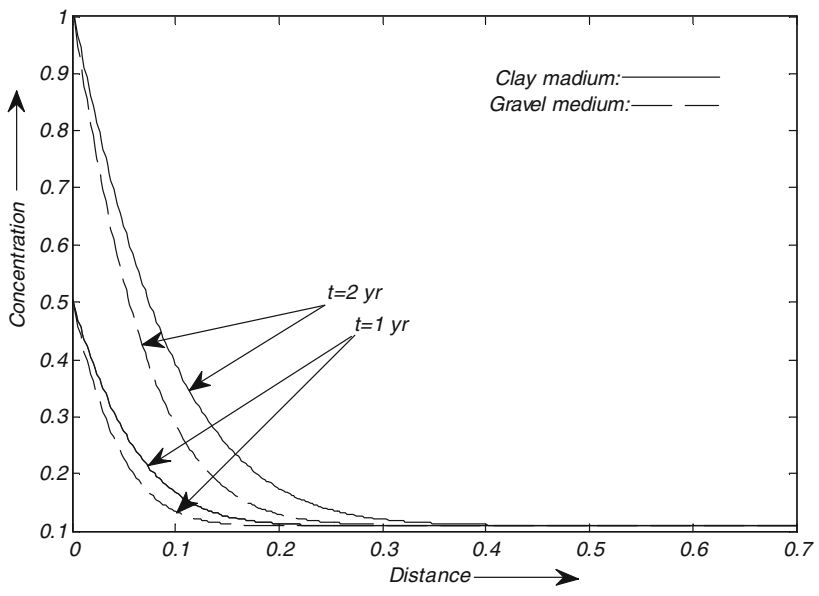

Figure 10. Concentration distribution for exponentially decreasing velocity pattern for boundary condition $\frac{\gamma}{U} t$.

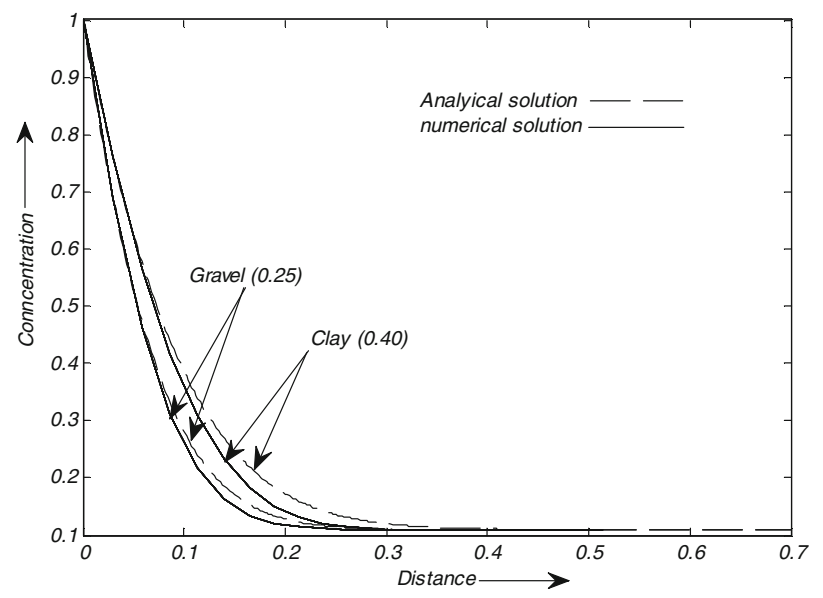

Figure 11. Comparison of concentration distribution for sinusoidal velocity pattern for boundary condition $\frac{\gamma}{U} t$.

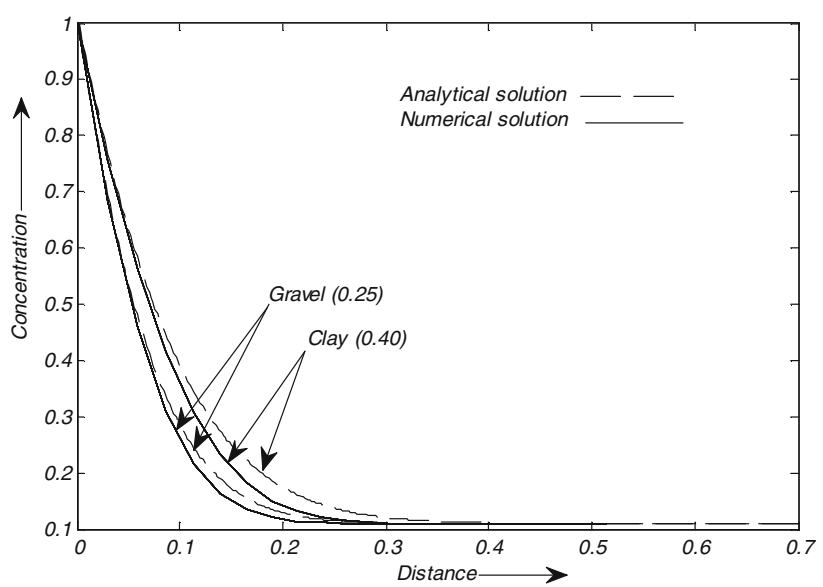

Figure 12. Comparison of concentration distribution for exponentially decreasing velocity pattern for boundary condition $\frac{\gamma}{U} t$.

concentration pattern for the different boundary conditions in Eqs. (5), (21) and (26) are predicted in figure 13 for the exponential decreasing velocity pattern. The concentration 


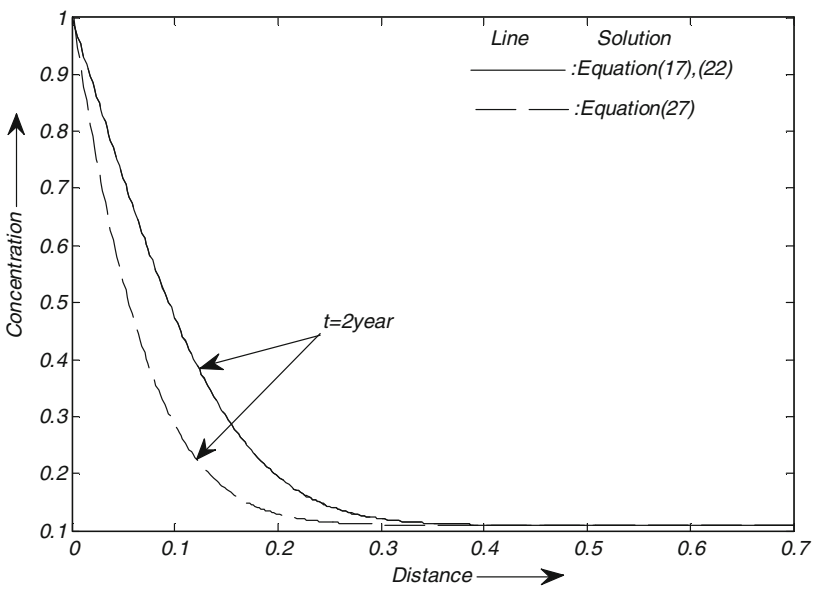

Figure 13. Comparison of concentration distribution for exponentially decreasing velocity pattern for different boundary conditions with gravel medium.

value for the boundary condition in Eqs. (5) and (21) attains the same concentration value at each position. It attains the higher value of concentration as compared to the boundary condition in Eq. (26). The surface concentration pattern for the sinusoidal and exponentially decreasing velocity pattern is predicted in figures 14 and 15 . It is observed form these figures that concentration values increase with respect to time but decrease with respect to distance.

The Relative error is used to measure the accuracy of the numerical solution against analytical one. In numerical solution, two parameters $\Delta T$ and $\Delta Z$ are necessary to investigate the effect of changes. The accuracy of the solution is investigated through relative error analysis evaluated in tables 1,2 and 3. The Relative error for the different boundary conditions along with gravel medium is given in tables 1,2 and 3. Tables 1, 2 and 3 show the relative error for the boundary condition defined in Eqs. (5), (21) and (26), respectively, for the time period of

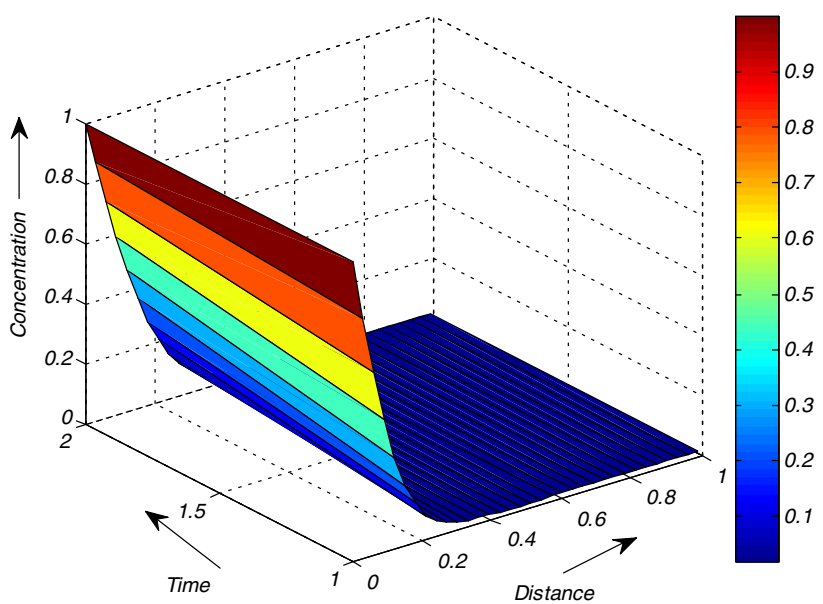

Figure 14. Surface concentration distribution for sinusoidal velocity pattern for boundary condition $\frac{c_{0}}{2}(1+\sec w t)$.

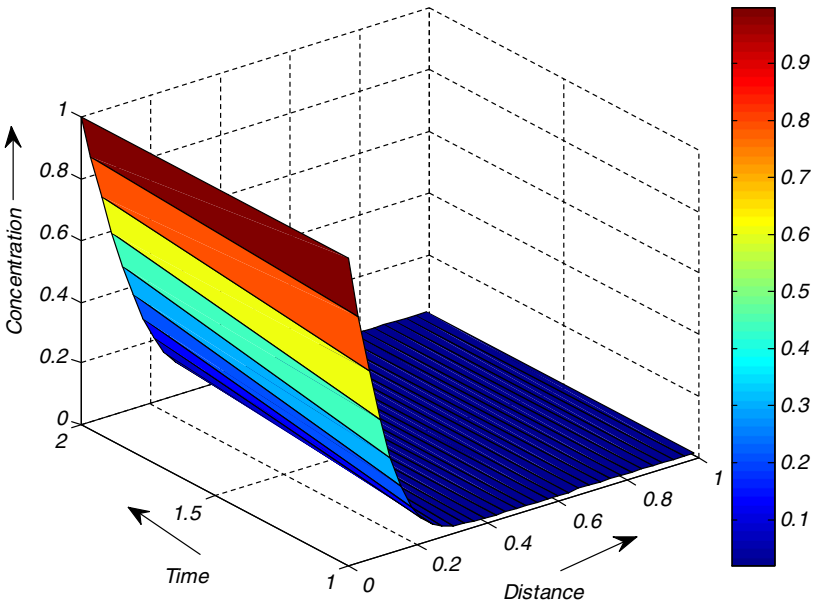

Figure 15. Surface concentration distribution for exponentially decreasing velocity pattern for boundary condition $\frac{c_{0}}{2}(1+\sec w t)$.

Table 1. Relative error for gravel medium at particular 2 year for boundary condition $\frac{c_{0}}{2}(1+\sec w t)$.

\begin{tabular}{lccc}
\hline $\begin{array}{l}\text { Distance } \\
(\mathrm{X})\end{array}$ & $\begin{array}{c}\text { Analytical } \\
\text { solution }\end{array}$ & $\begin{array}{c}\text { Numerical solution } \\
\Delta T=0.001\end{array}$ & $\begin{array}{c}\text { Relative } \\
\text { error (RE) }\end{array}$ \\
\hline 0.15 & 0.3106 & 0.3787 & 0.2192 \\
0.30 & 0.1228 & 0.1211 & 0.0138 \\
0.45 & 0.1100 & 0.1100 & 0.0000 \\
\hline
\end{tabular}

Table 2. Relative error for gravel medium at particular 2 year for boundary condition $(1-w U t)^{\frac{q}{w}}$.

\begin{tabular}{lccc}
\hline $\begin{array}{l}\text { Distance } \\
(X)\end{array}$ & $\begin{array}{c}\text { Analytical } \\
\text { Solution }\end{array}$ & $\begin{array}{c}\text { Numerical solution } \\
\Delta T=0.001\end{array}$ & $\begin{array}{c}\text { Relative } \\
\text { error (RE) }\end{array}$ \\
\hline 0.15 & 0.3105 & 0.3785 & 0.2190 \\
0.30 & 0.1228 & 0.1211 & 0.0138 \\
0.45 & 0.1101 & 0.1100 & 0.0009 \\
\hline
\end{tabular}

Table 3. Relative error for gravel medium at particular 2 year for boundary condition $\frac{\gamma}{U} t$.

\begin{tabular}{lccc}
\hline $\begin{array}{l}\text { Distance } \\
(X)\end{array}$ & $\begin{array}{c}\text { Analytical } \\
\text { solution }\end{array}$ & $\begin{array}{c}\text { Numerical solution } \\
\Delta T=0.001\end{array}$ & $\begin{array}{c}\text { Relative } \\
\text { error (RE) }\end{array}$ \\
\hline 0.15 & 0.1781 & 0.2139 & 0.2010 \\
0.30 & 0.1113 & 0.1111 & 0.0017 \\
0.45 & 0.1100 & 0.1100 & 0.0001 \\
\hline
\end{tabular}

2 years along with minimum range of porosity for gravel medium. The relative error decreases as distance increases in tables 1,2 and 3. From these tables, we observed that the maximum relative error is attained for the boundary condition defined in Eq. (5) as compared to other.

The validation of the model equation shown in the existing research work has been performed using Gharehbaghi [14] and van Genuchten [21]. The different inputs are for the validation purpose are shown in table 4 . The 
Table 4. Different input for the validation purpose.

\begin{tabular}{lcccc}
\hline $\begin{array}{l}\text { Author's name /Input } \Rightarrow \\
\Downarrow\end{array}$ & Source concentration $\left(\mathrm{c}_{0}\right)$ & Retardation factor $(R)$ & Dispersion coefficient $\left(D_{0}\right)$ & Seepage velocity $\left(U_{0}\right)$ \\
\hline (I) Author's input & 1 & 8.5 & 0.03 & 0.5 \\
(II) Gharehbaghi [14] & 1 & - & 0.71 & 0.60 \\
(III) van Genuchten [21] & 1 & 3 & - & 0.91 \\
\hline
\end{tabular}

concentration distribution pattern for different inputs predicted for the exponential decreasing velocity pattern with minimum range of porosity of gravel medium is shown in figure 16. From this figure, we observed that the concentration value for the input (1) attains the minimum level of concentration as compared to the inputs (2) and (3). Beyond some distance, input (1) and input (2) attain the same value of concentration but input (3) attains little higher concentration value as compared to the input (1) and input (2). At the end of the domain, both the inputs attain their minimum level of concentration values.

In solute transport modelling, various parameters play an important role in predicting the contaminant concentration distribution. In this study, sensitivity analysis has been described as a mean of determining the model input parameters that exert the strongest influence on calculated concentration distribution profile. The concentration distribution patterns for the different values of seepage velocity, dispersion coefficient and the distribution coefficient in the exponential decreasing velocity pattern are shown in the table 5a-c, respectively. Similarly, the sinusoidal varying velocity patterns are shown in table $6 \mathrm{a}-\mathrm{c}$ respectively. As shown in tables $5 \mathrm{a}$ and $6 \mathrm{a}$ the concentration values decrease with increasing seepage velocity with their respective distance. The concentration value attains its minimum level for the sinusoidal varying velocity pattern as compared to the exponential decreasing velocity pattern. The maximum concentration values attains for increasing

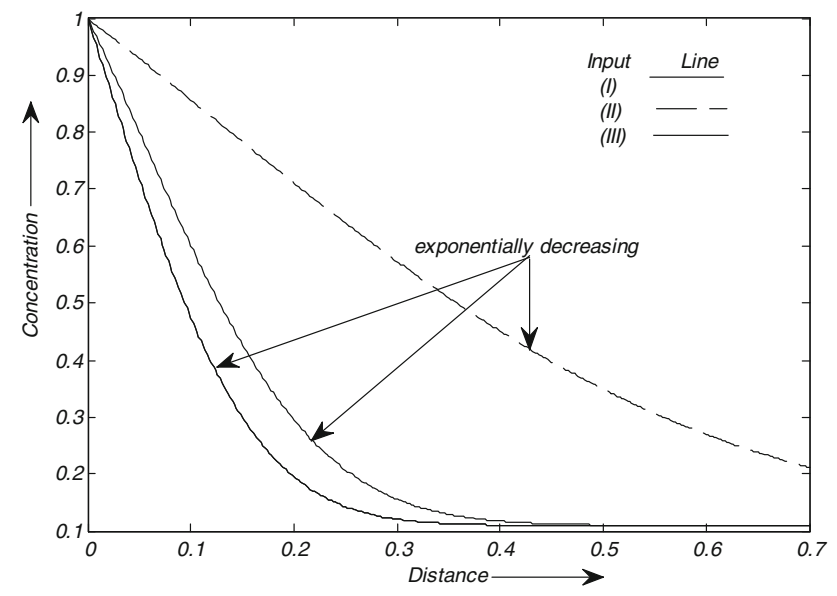

Figure 16. Concentration distribution for exponentially decreasing velocity pattern for boundary condition $\frac{c_{0}}{2}(1+\sec w t)$ with different inputs.
Table 5. The concentration distributions values for the different values.

Seepage velocity $\left(U_{0}\right) /$

distance $(x) \Rightarrow \quad 0.1 \quad 0.2 \quad 0.3 \quad 0.4$

(a) Seepage velocity in exponential decreasing velocity pattern

\begin{tabular}{lllll}
\hline 0.3 & 0.4739 & 0.1949 & 0.1206 & 0.1107 \\
0.5 & 0.4691 & 0.1927 & 0.1202 & 0.1107 \\
0.7 & 0.4671 & 0.1917 & 0.1200 & 0.1106 \\
\hline
\end{tabular}

Dispersion coefficient $\left(D_{0}\right) /$

distance $(x) \Rightarrow \quad 0.1 \quad 0.2 \quad 0.3 \quad 0.4$

(b) Dispersion coefficient in exponential decreasing velocity pattern

\begin{tabular}{lllll}
\hline 0.01 & 0.2328 & 0.1130 & 0.1100 & 0.1100 \\
0.03 & 0.4691 & 0.1927 & 0.1202 & 0.1107 \\
0.05 & 0.5735 & 0.2842 & 0.1557 & 0.1182 \\
\hline
\end{tabular}

Distribution coefficient $\left(k_{d}\right) /$

distance $(x) \Rightarrow \quad 0.1 \quad 0.2 \quad 0.3 \quad 0.4$

(c) Distribution coefficient in exponential decreasing velocity pattern

\begin{tabular}{lllll}
\hline 0.5 & 0.6956 & 0.4404 & 0.2677 & 0.1730 \\
2.5 & 0.4691 & 0.1927 & 0.1202 & 0.1107 \\
5.5 & 0.3122 & 0.1237 & 0.1102 & 0.1100 \\
\hline
\end{tabular}

values of dispersion coefficient with their respective travel distance observed from tables $5 \mathrm{~b}$ and $6 \mathrm{~b}$. Similarly, with increasing the value of distribution coefficient the concentration values also increased with their respective distance observed from tables $5 \mathrm{c}$ and $6 \mathrm{c}$. The concentration value follows its decreasing pattern with respect to the travel distance in each of the tables 5a and $6 \mathrm{c}$.

\section{Summary and conclusion}

This present paper provided an analytical and numerical solution for solute transport model equation in homogeneous semi-infinite porous media. The solution for onedimensional solute transport modelling in solid liquid interphase is obtained with the concept of linear isotherms. The various time dependent boundary conditions are taken into consideration for the solution of the problem. The 
Table 6. The concentration distributions values for the different values.

Seepage velocity $\left(U_{0}\right)$ /

distance $(x) \Rightarrow \quad 0.1 \quad 0.2 \quad 0.3 \quad 0.4$

(a) Seepage velocity in sinusoidal velocity pattern

\begin{tabular}{lllll}
\hline 0.3 & 0.4716 & 0.1933 & 0.1202 & 0.1106 \\
0.5 & 0.4669 & 0.1911 & 0.1198 & 0.1106 \\
0.7 & 0.4648 & 0.1902 & 0.1197 & 0.1106 \\
\hline
\end{tabular}

Dispersion coefficient $\left(D_{0}\right) /$

$\begin{array}{lllll}\text { distance }(x) \Rightarrow & 0.1 & 0.2 & 0.3 & 0.4\end{array}$

(b) Dispersion coefficient in sinusoidal velocity pattern

\begin{tabular}{lllll}
\hline 0.01 & 0.2362 & 0.1129 & 0.1100 & 0.1100 \\
0.03 & 0.4669 & 0.1911 & 0.1198 & 0.1106 \\
0.05 & 0.5715 & 0.2821 & 0.1546 & 0.1178
\end{tabular}

Distribution coefficient $\left(k_{d}\right) /$

$\begin{array}{lllll}\text { distance }(x) \Rightarrow & 0.1 & 0.2 & 0.3 & 0.4\end{array}$

(c) Distribution coefficient in sinusoidal velocity pattern

\begin{tabular}{lllll}
\hline 0.5 & 0.6940 & 0.4381 & 0.2656 & 0.1716 \\
2.5 & 0.4669 & 0.1911 & 0.1198 & 0.1106 \\
5.5 & 0.3101 & 0.1232 & 0.1102 & 0.1100 \\
\hline
\end{tabular}

effects of decay constant and zero order production term are considered. The derived solution is validated with the research work done by Gharehbaghi [14] and van Genuchten [21]. The result may be useful as a preliminary predictive tools for groundwater resource and management. This study will be further explored for distributed pollutant source concentration. The following conclusions are drawn:

1. The Laplace Transform Technique and Finite Difference Scheme are used for analytical and numerical solutions, respectively and are in good agreement.

2. Accuracy of the numerical solution against analytical solution is explored by relative error analysis and the relative error is found to be 0.2 .

3. Two different velocity patterns are used to predict the concentration distribution profile and observed that the contaminant concentration value is high in clay medium as compared to the gravel medium.

\section{Acknowledgements}

The authors are thankful to the reviewers whose constructive comments helped improve the quality of the paper. The authors are also thankful to IIT(ISM) Dhanbad, for providing financial support to $\mathrm{Ph}$.D. candidate under the JRF scheme. This work was supported by the CSIR Project no. 25(0251)/16/EMR-II.

\section{Appendix}

Using Eq. (16) in Eq. (13) and the corresponding initial and boundary conditions given in Eq. (14)-(15b), we have the following equations:

$$
\begin{gathered}
R \frac{\partial k}{\partial T}=D_{0} \frac{\partial^{2} k}{\partial z^{2}} \\
k(z, 0)=\left(c_{i}+w\right) \exp \left(-\frac{u_{0} z}{2 D_{0}}\right) ; \quad z>0, T=0 \\
k(0, T)=\frac{c_{0}}{4}\left(4+w^{2} T^{2}\right) \exp \left(\frac{u_{0}^{2} T}{4 R D_{0}}\right) ; \quad z=0, T>0 \\
\frac{\partial k}{\partial z}=-\frac{u_{0} k}{2 D_{0}} ; \quad z \rightarrow \infty, T>0 .
\end{gathered}
$$

After applying the Laplace Transform Technique (LTT) in Eq. (46)-(49), we get the desired solution of $\bar{k}(z, P)$ written as follows:

$$
\begin{aligned}
\bar{k}(z, P)= & C_{1} \exp \left(-\sqrt{\frac{R P}{D_{0}}} z\right)+C_{2} \exp \left(\sqrt{\left.\frac{R P}{D_{0}} z\right)}\right. \\
& +\frac{c_{i}+w}{P-\alpha^{2}} \exp \left(-\frac{u_{0} z}{2 D_{0}}\right)
\end{aligned}
$$

where; $\bar{k}(z, P)=\int_{0}^{\infty} k(z, T) e^{-P T} d T$.

After using the boundary conditions of Eqs. (48) and (49) in Eq. (50). We get

$$
C_{2}=0
$$

and $\quad C_{1}=\frac{c_{0}}{2}\left(\frac{2}{P-\alpha^{2}}+\frac{w^{2}}{\left(P-\alpha^{2}\right)^{3}}\right)-\frac{c_{i}+w}{P-\alpha^{2}}$.

Using the values of $C_{1}$ and $C_{2}$ from Eq. (51) and (52) in Eq. (50), we have

$$
\begin{aligned}
\bar{k}(z, P)= & \left(\frac{c_{0}-c_{i}-w}{P-\alpha^{2}}\right) \exp \left(-\sqrt{\frac{R P}{D_{0}}} z\right) \\
& +\frac{c_{0} w^{2}}{2\left(P-\alpha^{2}\right)^{3}} \exp \left(-\sqrt{\frac{R P}{D_{0}}} z\right) \\
& +\frac{c_{i}+w}{P-\alpha^{2}} \exp \left(-\frac{u_{0} z}{2 D_{0}}\right) .
\end{aligned}
$$

Now taking the inverse Laplace Transform of Eq. (53) the solution of $k(z, T)$ can be written as follows:

$$
\begin{aligned}
k(z, T)= & \left(\frac{c_{0}-c_{i}-w}{2}\right) F_{11}(z, T)+\frac{w^{2} c_{0}}{2} G_{11}(z, T) \\
& +H_{11}(z, T)
\end{aligned}
$$

where 


$$
\begin{aligned}
F_{11}(z, T)= & \exp \left(\frac{u_{0}^{2} T}{4 R D_{0}}-\frac{u_{0} z}{2 D_{0}}\right) \operatorname{erfc}\left(\frac{z R-u_{0} T}{2 \sqrt{D_{0} R T}}\right) \\
& +\exp \left(\frac{u_{0}^{2} T}{4 R D_{0}}+\frac{u_{0} z}{2 D_{0}}\right) \operatorname{erfc}\left(\frac{z R+u_{0} T}{2 \sqrt{D_{0} R T}}\right) \\
G_{11}(z, T)= & \left(\frac{1}{2 \alpha}\right)\left(\alpha T^{2}-\frac{z T}{2} \sqrt{\frac{R}{D_{0}}}+\frac{z}{4 \alpha^{2}}-\frac{z T}{2}+\frac{z^{2}}{4 \alpha} \sqrt{\frac{R}{D_{0}}}\right) \\
& \times \exp \left(\frac{u_{0}^{2} T}{4 R D_{0}}-\frac{u_{0} z}{2 D_{0}}\right) \operatorname{erfc}\left(\frac{z R-u_{0} T}{2 \sqrt{D_{0} R T}}\right) \\
+ & \left(\frac{1}{2 \alpha}\right)\left(\alpha T^{2}+\frac{z T}{2} \sqrt{\frac{R}{D_{0}}}-\frac{z}{4 \alpha^{2}}+\frac{z T}{2}-\frac{z^{2}}{4 \alpha} \sqrt{\frac{R}{D_{0}}}\right) \\
& \times \exp \left(\frac{u_{0}^{2} T}{4 R D_{0}}+\frac{u_{0} z}{2 D_{0}}\right) \operatorname{erfc}\left(\frac{z R+u_{0} T}{2 \sqrt{D_{0} R T}}\right) \\
& -\left(\frac{1}{2 \alpha}\right) \frac{z}{\alpha} \sqrt{\frac{T}{\pi}} \mathrm{e}^{-\frac{z^{2} R}{4 D_{0}}}
\end{aligned}
$$

$$
H_{11}(z, T)=\left(c_{i}+w\right) \exp \left(\frac{u_{0} z}{2 D_{0}}-\frac{u_{0}^{2} T}{4 R D_{0}}\right) .
$$

Using Eq. (54) in Eq. (16) we get the complete desired solution given in Eq. (17).

On applying the same procedure we get the desired analytical solutions for the conditions in Eqs. (21) and(26) given in Eqs. (22) and (27).

\section{References}

[1] Kumar N 1983 Unsteady flow against dispersion in finite porous media. J. Hydrol. 63(3): 345-358

[2] Van Kooten J J A 1996 A method to solve the advectiondispersion equation with a kinetic adsorption isotherm. $A d v$. Water Resour. 19(4): 193-206

[3] Leij F J, Priesack E and Schaap M G 2000 Solute transport modeled with Green's functions with application to persistent solute sources. J. Contam. Hydrol. 41(1): 155-173

[4] Zheng C and Bennett G D 2002 Applied contaminant transport modeling. New York: Wiley-Interscience

[5] Quezada C R, Clement T P and Lee K K 2004 Generalized solution to multi-dimensional multi-species transport equations coupled with a first-order reaction network involving distinct retardation factors. Adv. Water Resour. 27(5): 507-520

[6] De Smedt F, Brevis W and Debels P 2005 Analytical solution for solute transport resulting from instantaneous injection in streams with transient storage. J. Hydrol. 315(1): 25-39
[7] Zhan H, Wen Z, Huang G and Sun D 2009 Analytical solution of two-dimensional solute transport in an aquiferaquitard system. J. Contam. Hydrol. 107(3): 162-174

[8] Qiu Y, Deng B and Kim C N 2010 Analytical solution for spatially dependent solute transport in streams with storage zone. J. Hydrol. Eng. 16(8): 689-694

[9] Gao G, Zhan H, Feng S, Fu B and Huang G 2012 A mobileimmobile model with an asymptotic scale-dependent dispersion function. J. Hydrol. 424: 172-183

[10] You K and Zhan H 2013 New solutions for solute transport in a finite column with distance-dependent dispersivities and time-dependent solute sources. J. Hydrol. 487: 87-97

[11] Deng B, Li J, Zhang B and Li N 2014 Integral transform solution for solute transport in multi-layered porous media with the implicit treatment of the interface conditions and arbitrary boundary conditions. J. Hydrol. 517: $566-573$

[12] Singh M K and Das P 2015 Scale dependent solute dispersion with linear isotherm in heterogeneous medium. $J$. Hydrol. 520: 289-299

[13] Majdalani S, Chazarin, J P, Delenne C and Guinot V 2015 Solute transport in periodical heterogeneous porous media: lmportance of observation scale and experimental sampling. J. Hydrol. 520: 52-60

[14] Gharehbaghi A 2016 Explicit and implicit forms of differential quadrature method for advection-diffusion equation with variable coefficients in semi-infinite domain. J. Hydrol. 541: 935-940

[15] Sanskrityayn A and Kumar N 2016 Analytical solution of advection-diffusion equation in heterogeneous infinite medium using Green's function method. J. Earth Syst. Sci. 125(8): 1713-1723. https://doi.org/10.1007/s12040-0160756-0

[16] Falta R W and Wang W 2017 A semi-analytical method for simulating matrix diffusion in numerical transport models. $J$. Contam. Hydrol. 197: 39-49

[17] Batu V 2006 Applied flow and solute transport modeling in aquifers: fundamental principles and analytical and numerical methods. Boca Raton, FL: CRC

[18] Elfeki A M, Uffink G J and Lebreton S 2007 Simulation of solute transport under oscillating groundwater flow in homogeneous aquifers. J. Hydraul. Res. 45(2): 254-260

[19] Kumar A, Jaiswal D K and Kumar N 2010 Analytical solutions to one-dimensional advection-diffusion equation with variable coefficients in semi-infinite media. J. Hydrol. 380: 330-337

[20] Chen C, Liu F and Burrage K 2008 Finite difference methods and a Fourier analysis for the fractional reaction-subdiffusion equation. Appl. Math. Comput. 198: 754-769

[21] Van Genuchten M T 1981 Analytical solutions for chemical transport with simultaneous adsorption, zero-order production and first-order decay. J. Hydrol. 49(3): 213-233 